\title{
Sharing Map Annotations in Small Groups: $X$ Marks the Spot
}

\author{
Ben Congleton $^{1, *}$, Jacqueline Cerretani ${ }^{1, *}$, Mark W. Newman ${ }^{1,2}$, \\ and Mark S. Ackerman ${ }^{1,2}$ \\ ${ }^{1}$ School of Information \\ ${ }^{2}$ Department of Electrical Engineering and Computer Science \\ University of Michigan \\ 1075 Beal Avenue \\ Ann Arbor, MI 48109, USA \\ \{bcx, jackiejc, mwnewman, ackerm\} @umich. edu
}

\begin{abstract}
Advances in location-sensing technology, coupled with an increasingly pervasive wireless Internet, have made it possible (and increasingly easy) to access and share information with context of one's geospatial location. We conducted a four-phase study, with 27 students, to explore the practices surrounding the creation, interpretation and sharing of map annotations in specific social contexts. We found that annotation authors consider multiple factors when deciding how to annotate maps, including the perceived utility to the audience and how their contributions will reflect on the image they project to others. Consumers of annotations value the novelty of information, but must be convinced of the author's credibility. In this paper we describe our study, present the results, and discuss implications for the design of software for sharing map annotations.
\end{abstract}

Keywords: Map Annotation, Social Media, Sharing, Location Based Computing, HCI, Requirements Analysis.

\section{Introduction}

The boundary between "cyberspace" and physical space is eroding. Advances in location-sensing technology (e.g., GPS), coupled with an increasingly pervasive wireless Internet, have created a new class of applications where users create, share, and access location-based information linked seamlessly to their physical context.

Traditionally, when we think of connecting information to a geospatial location, we think in terms of maps. Although maps have been used for thousands of years, only recently have end users been able to create and annotate geo-spatially indexed information to easily share with others. Google Earth, Google Maps, Yahoo Maps, and Microsoft Live Maps allow one to render geospatial annotations, and also provide tools to create and add annotations directly on maps.

At present, these services are the most popular platforms for sharing and annotating geo-coded information. However, none were built from the ground up with the

* Co first authors.

T. Gross et al. (Eds.): INTERACT 2009, Part II, LNCS 5727, pp. 97-110. 2009.

(C) IFIP International Federation for Information Processing 2009 
intention of creating maps for sharing. In most cases, sharing annotated maps was a feature added on top of platforms primarily designed to help people navigate from place A to B or find local businesses. In this study we wanted to step back and explore the creation and sharing of annotated maps without the ingrained technological constraints of platforms that were built with other goals in mind.

Moreover, these systems have already begun to establish patterns of use that may discourage or preclude the wide range of applications we feel are possible and perhaps likely to emerge in this space. Geospatial annotation systems intended to support publicly shared annotations will necessarily be designed differently from those intended to support small intimate groups. Similarly, those intended to support large geographical areas will differ from those focused on specific communities. In order to illustrate this area and its potential more fully, we provide a brief sketch of three hypothetical applications involving spatial annotation in order to illustrate the potential diversity of applications within this category.

Orientator allows new university students to share the burden of exploring a new physical environment, while getting to know each other more rapidly. Groups, such as dormitory floormates or incoming $\mathrm{PhD}$ students in a large department, are tasked with annotating a shared map of their new campus. As each individual explores the area, they contribute to a shared representation of "interesting" and "useful" locations. Identities are linked to annotations, revealing participants interests and tastes to fellow students, facilitating the formation of personal relationships around shared interests.

PubCrawler supports the cataloging and annotation of a particular location type within a geographic area-i.e., places to drink. The community of users is defined by mutual interest rather than membership in an organization, and may be subdivided by more narrow interests within the topic (e.g., martini vs. microbrew enthusiasts). Selfappointed domain experts as well as less specialized community members select, rate, and annotate pubs and bars, and the shared record is used by all members to identify new places to explore.

LocalExpert allows users in a particular geographic area to pose queries to anonymous domain "experts" and receive replies from those experts or from a cache of previous responses. This application can be seen as a geospatial instance of Answer Garden [1], in which the determination of expertise is generated by privacy-aware automated location traces of users' movements and/or manual annotations of favorite locations. Queries might be specified using natural language (e.g., "Which store in Manhattan has the best selection of shoes?") or by identifying points on a map (e.g., "What does [Café Zola; 112 Washington St.] serve for lunch?").

Each of these applications shares a set of features: users must be able to indicate (either explicitly or implicitly) locations of interest, and other users must be able to "read" (again explicitly or implicitly) the annotations, opinions, and experiences of other users in order to form a judgment about the location described, the author of the annotation, or both.

Starting from this sketch of the design space, our study set out to characterize the design requirements for systems that supports shared geospatial annotations, and the choices and tradeoffs that designers of this emerging class of applications face. In particular we sought to address the following questions:

- How do people choose to annotate spatial locations on a map?

- How do users assess other individuals' map annotations? 
- How does the association of an individual with a map effect the interpretation of the annotations?

- What tools and affordances will best support both the authors and the audience for geospatial annotations?

The goal of our study was to gain insight into these questions. We examined the map annotation and interpretation behavior of 27 participants over a variety of conditions. We used the creation and act of sharing annotated maps as an analytic probe to better understand the processes used to annotate and interpret shared maps.

The paper proceeds as follows. First we lay out the design space for map annotations, followed with a brief review of the pertinent literature on this topic. We then describe the study, followed by the detailed findings. We finish with the design implications suggested by the study findings.

\section{Related Work}

Although there is considerable commercial interest in place-based annotations (e.g., the aforementioned Google Maps, Yahoo Maps, and Microsoft Live Maps), including recent developments focusing on shared annotations (e.g., BriteKite, Loopt, and Pla$\operatorname{tial}^{1}$ ), relatively little research attention has been paid to systems for sharing mapbased annotations. Here we detail the research literature that does exist and briefly discuss additional literature that touches on this subject.

\section{Studies of Social Map Annotation}

A small number of studies examine social applications using maps. First, MapChat and MapMail $[6,13]$ are recent examples that attempt to extend the static nature of maps, using them to geographically ground real-time and asynchronous conversations, respectively. MapChat focuses on grounding conversation on place, rather than the creation of a persistent information artifact, which will be central to our study. MapMail primarily visualizes email information on a map.

Second, Ludford and others [10] studied the use of user-created, location-based reminders (LBR) and place bookmarks, as a method of capturing place metadata. They evaluated participants sharing fictitious personal bookmarks and location reminders on a prototype system. Their participants shared content created for personal purposes, rather than creating content to be explicitly shared.

Third, Priedhorsky and Terveen [14] built a geowiki, a new platform for building community maps. Their work has, to date, focused on the implementation of the geowiki, rather than on how users annotate and interpret specific information on community-created maps. A brief evaluation notes that users found the annotations and comments on the geowiki useful.

Fourth, Jones et al. [9] gathered design requirements for location-aware community systems. In interviews, they found that users were interested in having access to user contributed annotations of place (i.e comments and ratings). Users were also interested in creating their own annotations, but only if they were shared anonymously or with strict access controls. Their work does not discuss the act of creating or interpretation of these annotations.

1 http://britekite.com, http://www.loopt.com, http://www.platial.com 
Finally, two other streams of locative research relate to social maps: the real-time sharing of location [3, 15], and annotations accessible only at specific locations [4, 7] (i.e. E-graffiti) . Studies of active location sharing systems are primarily focused on mutual awareness of location, rather than static annotations. Studies of systems such as E-graffiti have helped us understand the use of place-specific notes through real world deployments, but provide little exploration of the practices surrounding annotation.

\section{Studies of Annotation More Broadly}

A range of studies examines annotation in general. For example, Marshall [11] discusses dimensions of annotation of particular interest to our work: formal v. informal, explicit v. tacit (explicit annotations are easily understood by wide audiences, whereas tacit annotations may require specific context from the author), published v. private, and type of audience (global, institutional, workgroup, or personal). Our study explores the informal annotation of maps, where annotations are published and shared within small groups. Marshall [12] explores the relationship between personal and public annotations in hypertext, noting substantial refinement of personal annotations before they were shared online.

A number of other studies examine the technical features of and user motivations for using annotation systems. For example, Carter et al. [5] in their study of PDAbased annotations on shared multimedia content, characterize three categories of annotation: annotations for personal use, collaborative annotations, and public/social annotations. Ames and Naaman [2] describe four categories of motivation for adding tags to personal content on a photo-sharing site: personal-organization, personalmemory, social-organization, and social-communication. They found that participants who are motivated to share contextual information about photos socially often annotate with a specific known audience in mind.

Taken as a whole, these previous studies have elucidated the range of technical support required for annotation systems and the motivations for sharing, and have done so for a geospatial context. None, to our knowledge, have taken a close look at how people choose place-based information to share with others in a social context. This was the goal of our study. In the next section we describe our study, its methods, and the data collection.

\section{Methods}

To better understand how individuals annotate, interpret, and socialize around shared maps we designed a 4-phase study involving twenty-seven masters and $\mathrm{PhD}$ students (12 women and 15 men). They had lived in Ann Arbor, Michigan (USA), from 1 week to 30 years.

The four phases were designed with the following goals in mind. Phase 1: understand the uninfluenced annotation habits of students, paying particular attention to the types of annotations that they made. Phase 2: understand how users create maps after they have been encouraged to interpret the maps of others. Phase 3: understand how participants create maps to be shared with others when their identity is known. Phase 4: understand the role the shared maps could play in the social interactions of a small, known group. 
Table 1. Our study consisted of four phases, in which participants created and/or viewed others' maps. Each participant was interviewed about their experiences creating and viewing the maps. (*: the same participants participated in phases 3 and 4 ).

\begin{tabular}{ccccccc}
\hline Phase & Participants & Viewed Maps & Annotated Map & Interviewed & Audience \\
\hline 1 & 11 & & $\mathrm{X}$ & $\mathrm{X}$ & Lounge \\
\hline 2 & 6 & $\mathrm{X}$ & $\mathrm{X}$ & $\mathrm{X}$ & Lounge \\
\hline 3 & $10^{*}$ & $\mathrm{X}$ & $\mathrm{X}$ & $\mathrm{X}$ & Group \\
\hline 4 & $10^{*}$ & $\mathrm{X}$ & & $\mathrm{X}$ & Group \\
\hline
\end{tabular}

In phase 1, eleven participants were given markers and pens and asked to annotate a packet of 8.5"x11" (21.59 cm x $27.94 \mathrm{~cm})$ paper maps (Fig. 1), showing various sections of Ann Arbor at different zoom levels. They were told the content of the maps would be posted publicly in the student lounge and shared with new students in their department, and that their name might or might not be included on their map.

In phase 2, six participants were presented serially with three or more annotated map packets from phase 1 , then asked to annotate their own map packet, with the same tools and information about attribution given to phase 1 participants.

Phases 3 and 4 used the same participants: a group of 10 masters students who knew each other and had all participated in the same series of design workshops during the previous two months. In phase 3, they were shown up to three annotated map packets from phase 2, then asked to annotate their own map: a larger, 22x17" (55.88 $\mathrm{cm} \times 43.18 \mathrm{~cm}$ ) sheet with a close up of Ann Arbor's downtown and university areas on the front, and a bird's-eye view of the entire city on the back. Participants were asked to write their name on the maps, and were told that the maps would be distributed as a packet, with attribution, to the other members of this phase.

Phase 4 was a three week period immediately following phase 3 during which participants were in possession of the map packets (the packet lacked one phase 3 participant's map due to a technical issue). During the three weeks, they received two short email questionnaires about their use of the maps and interactions with other phase 3/4 members. At the end of the three weeks, participants were again interviewed about their experiences with the maps and each other, and were asked to compare two of their fellow group member's paper maps side-by-side with a duplicate created in Google Maps.

We interviewed each participant in each phase of the study. In phases where maps were created (1-3), participants were asked about decisions made during map creation. In phases where participants viewed the annotated maps of others (2-4), they were asked to discuss what they found interesting about the maps and their annotators. During analysis, we coded the 27 annotated maps, the 37 transcribed interviews, and the two rounds of email questionnaires. We paid particular attention to the process and strategies involved in annotation of the maps, interpretation of the maps, and the role maps played in one-to-one and group interactions in the Phase 3 and 4 participants. 


\section{Results}

As noted, all study participants created maps. In addition, phase 2-4 participants both looked at and created maps. Moreover, phase 3 and 4 participants created maps for a known social group and reflected on the maps created by other group members. Thus, our findings fall into three distinct but related categories:

* Map Annotation: How did participants choose to annotate their maps? What content did they include and omit, and how did they choose to indicate elements of interest?

* Map Interpretation: How did participants make sense of other participants' maps? What aspects did they find interesting and valuable, and what aspects were viewed negatively?

* Impacts on Interpersonal Relationships: What effect did sharing maps have on the relationships among members of a known social group? Did opinions of other group members change as a result of viewing others' maps?

In this section, we report our observations related to each category.

\subsection{Annotating Maps}

When annotating maps (Figure 1), participants were faced with choices about what items to include on the map, what to say about each item, and how to graphically represent the annotations on the map. In making these decisions, participants considered both the usefulness of the map for their audience, and how it reflected upon them personally.

An implicit concern among participants was to include annotations that were useful to the perceived audience. In the interviews, it appeared that authors considered their experience with a location and whether it would be novel and interesting to the audience, and sought to balance what they had to offer with what they thought the reader would want to see. In addition, a few authors sought to transform some aspect of the reader's experience of the area being annotated. As a secondary concern, authors used their annotation choices to attempt to manage others' impressions of them.

\section{Finding: Participants annotated based on personal experience}

Participants mainly chose to share locations they had experience with. Some explained that by including a location, they felt they were endorsing it, and thus should be able to substantiate that choice with experience. For example:

I wouldn't want to put something on the map that I've never been to. (p2.02) ${ }^{2}$

The emphasis on personal experience was evident in one participant's decision to omit a jewelry shop that she didn't view as "hers."

There was a jewelry shop that I thought about putting on there.... I guess I feel like it's not really my place. My roommate likes to go there a lot, so I felt like it was more her place and not mine. (p1.09)

2 Participant quotes are identified by phase and participant number. "p1.09" refers to the 9th interviewee in Phase 1. Since the same individuals were interviewed in Phases 3 and 4, each participant's number remains the same across the two phases. Thus "p3.01" and "p4.01" refer to two different interviews with the same person. 


\section{Finding: Participants tried to anticipate the usefulness of their annotations}

The desire to be "useful" to one's audience generalized across all phases, but the construction of "usefulness" varied depending on the participants' perception of who the audience was. In phases 3 and 4, where the audience was known and familiar, participants often figured that novel content was the most likely to interest their audience. Novelty was expressed as both new locations and new information about familiar locations.

I tried to think of things that I thought were really...that I appreciated about Our City, rather than things that everybody would put, like little secrets that I know. (p2.05)

Indeed, in phase 3 and 4, these "little secrets" were common on the maps. Participants annotated such locations as a hidden, graffiti-covered alley, a running route through an area with interesting houses, and an unlikely study location in the natural history museum. One participant created a whole category for "Food Secrets and Wine."

More quotidian annotations were included in these phases as well, but most often with the intention of being useful. One participant marked several parking garages in the somewhat congested downtown area. Another marked all the coffee shops he knew had WIFI. Some marked parks good for weekend outings, or main roads in and out of the city.

Some phase 1 and 2 participants, who only knew their map would be shared in a public space, felt that the needs and interests of their audience were unclear or elusive. As a possible consequence, more reflective individualistic annotations were included on the maps in these phases, such as commuting routes, workplaces, private personal routines and places participants aspired to visit. Though this may be related to the fact that these were among the seed suggestions in the instructions for these phases, there is also some evidence that an author's lack of knowledge about his audience may lead to a more introspective map. Here, one participant explains his choice to include a marginally-relevant location:

I did think twice about whether I should put in the comic books and the game stores... I think most people wouldn't care where those are... I just put it down in case there was someone else who did care, who did want to see it. (p1.06)

Another participant expressed it in terms of the low consequences of disclosure:

I though about the surgery one a little bit, because if it's public with your name on it, and people you... I don't care if it's strangers, but if it's a lot of people I know, I may not want to explain it to everyone all the time. (p1.13)

Notably, even some participants in phases 3 and 4 felt that their notion of audience was still too vague to be directive. One participant expressed his confusion about whom to annotate for this way:

I'm looking at the maps... I might look for hospitals, maybe other people looking for schools, other people looking for restaurants. They're not all the same. (p4.05)

Thus, even though participants wanted to create content that was interesting and useful to others, they were not always able to assess whether choices they were making were going to be well received.

\section{Finding: Participants sought to have an actionable impact on their audience}

Beyond utility, some participants expressed the desire to have a certain effect on readers of their maps. One user expressed the desire to influence people towards certain behaviors: 


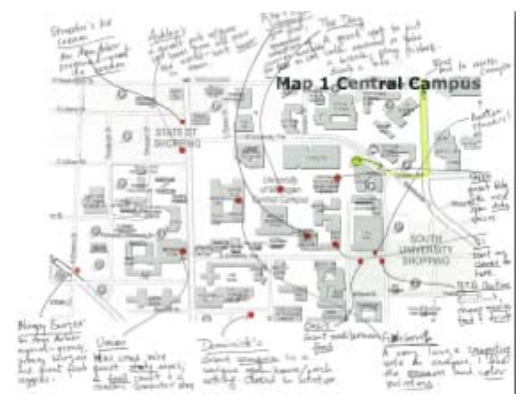

Fig. 1. Participants annotated paper maps with information they wanted to share with their peers

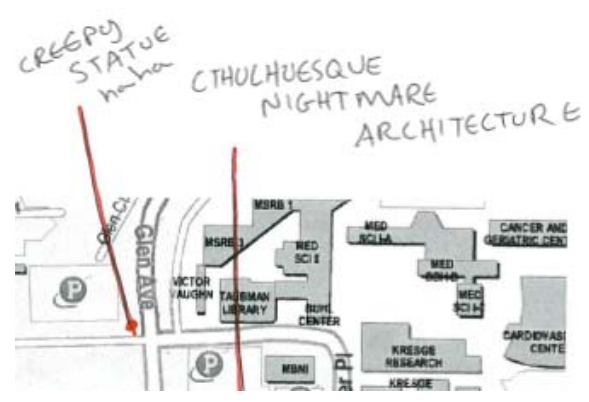

Fig. 2. Some participants labeled unusual locations in hopes to inspire certain emotions in their audience

I like encouraging people to do socially responsible things like shopping at the farmer's market, so that was definitely going to come out in this. (p3.03)

Another participant, whose choice of locations was unusual and annotation style concise but evocative (see Fig. 2), hoped to inspire certain emotions in his audience:

I would hope that people would recognize some of these landmarks, if you'll call them that, or be curious about certain places. (p3.06)

\section{Finding: "Face work" played a part in participants' choice of annotations}

While participants were more emphatic about the informational utility of their maps than the way the maps reflected their personality, "face work" [8] did emerge as part of some participants' filtering process and affected their annotation choices.

I was cognizant of who I was and what kind of persona I was putting out there. (p4.10) Novelty also played a part in some participants' expression of their personality.

I feel that I am revealing more by putting in things that aren't obvious. (p1.11)

As outlined above, novelty was not only expressed in the choice of points, but also in the explanations that accompanied them. One user explained how this related to her presentation of self:

The point is for people to find out something about you from your map... you have to include why you like that place, or why you should go there, instead of just pointing it out. (p2.01)

\subsection{Interpreting Map Annotations}

Not surprisingly, participants emphasized utility in their interpretation of others' map annotations. However, other factors came into play, such as assessing the author's similarity to themselves and credibility. Readers drew on many aspects of the annotations to assess their quality and personal relevance, including the content, style, level of detail, as well as their assessment of the author's personality and interests.

\section{Finding: Readers focused on usefulness in judging maps}

In keeping with their emphasis on creating informational value when annotating maps, participants judged others' maps most strongly on their usefulness, with a 
significant emphasis on novelty. In Phases 3 and 4, novel content was particularly valuable, in part because it was scarce:

I've been to all the places that they've circled. So, except for the studying place, I didn't

find it useful because I had already been to these places. (p3.10)

The quality of the explanations included in annotations was also considered important. Many participants felt that for an annotation to be useful, it needed to contain enough content for the reader to discern why the author endorsed that particular location. If the annotation contained more descriptive, informative details, it was valued more highly.

I noticed that a couple of people actually marked the Dana Building but one person has given more details - "it's a nice place to study, it's quiet, it has natural light"... I guess what I'm saying... there's a difference between marking it and giving more detail because you can decide if you would be interested in that place or not. (p4.07)

Participants explained that they used these details to help them profile the author's perspective and compare it to their own. This helped them judge if the map would be interesting or useful to them. This was particularly prevalent in cases where the reader did not know the author of the map.

Just the fact that there is more detail just makes me trust them more... if I circle thisAshley's-and say, "this place is good," I don't really know how or why they are evaluating it that way, but with the detail... there's something specific there that allows me to trust it. (p3.09)

\section{Finding: Readers tried to make sense of the author's personality}

In phase 4 , readers used their knowledge of the author's personality to help them evaluate the author's choice of points and decide if they were of value.

If I know the person prior and I kind of identify them, you know, it's going to add a little bit of credence to what they chose to show, or, like, it will probably increase the likelihood too that I will actually go to any of the places that they suggest or chose to mark. (p4.09)

When readers didn't know the author, and lacked prior knowledge of his or her personality, they often used annotations of familiar interests to calibrate their assessment.

I notice here that Blimpy Burger is marked and I approve. (p3.06)

In all the phases, when reviewing unattributed maps, participants looked for themes among the points to give them an idea of the author's character. This often resulted in an inventory of what the author included on the map, which was used to characterize the author lieu of more substantive knowledge about their personality. For example:

They mark the Arb for great hiking, walking or frisbee, so this leads me to think that this person has engaged in those activities (p2.06)

Less frequently, participants looked to the style and form of the annotations for indications of the character of an anonymous author.

The annotation influences your opinion of the person, I think. Like the second person had a very distinct handwriting. It was dark and legible. The third person kind of scrolled a little and they wrote very lightly. The first person seemed a little messy. (p3.07) 


\section{Finding: Readers valued aspects of informal annotations that improved informa- tion retrieval and effectively conveyed personality}

While form and style were used to discern an annotation author's character, they were more often judged by how well they supported information retrieval activities such as scanning and separating points from the substrate. One participant explains:

Color, labels followed by brief descriptions....an underlined title of each place with enough space to write it. Instead of on the map, it's on the border. I think this is a cleaner and improved way to represent these spots. (p3.06)

That said, form and style were substantially less important to our participants than the quality of the content. One participant summed up it up this way:

If it's sloppy but the content is good, I can work around the sloppiness. (p4.10)

However, there were cases where the free-form annotation capability allowed readers to form a better sense of the author's personality. In phase 4, the comparison between paper and Google Maps revealed that there was an intangible quality lost in the digitization of the paper maps. This was expressed even when participants felt the Google Map was more legible than the paper map. Most often, participants referred to the quality as "personality."

Just in terms of, like, being able to see somebody's handwriting and the colors they chose for, like, restaurant versus parking versus study rooms. I mean, there's just more personality, you know. Handwriting has a lot of personality in it. You can't convey that in Google

Maps, or a precise Google Map. (p3.09)

The last part of the participant's comment, "a precise Google Map," refers to the fact that most annotations on the paper maps were transferred to the Google Map in the form of points or specific routes. On the paper maps, more amorphous forms of annotation were frequently used, such as lines to represent parts of streets, shapes to indicate a general area, outlines around buildings, or visual textures (such as crosshatching) to represent a characteristic of a point or region. (See Figure 3.)

Another perceived advantage of paper maps over their Google Maps counterparts was that the paper allowed a comprehensive view of annotations and their content in a single glance. This is largely because only one complete textual annotation can be viewed at a time in Google Maps - other annotations appear as a list in a side panel, but are not directly linked to their points of reference. This ability to take in an annotation author's entire map at once was seen as valuable by participants.

I kind of feel like the digital one is a little bit less immediately accessible because all of [p3.10]'s annotations are available immediately [on the paper map] whereas with [the Google Map] I guess I'd have to go through each one and click on it individually. (p4.04)

\subsection{Effects of Map Sharing on Members of a Known Social Group}

\section{Finding: Maps yielded little new information about peers to members of a known group}

While phase 3 and 4 participants knew each other, and received a copy of most other phase 3 and 4 participants' maps, they felt that the maps did not enhance sense of belonging to a group. They also felt the maps revealed little, if anything, about the character of the group; when prompted, participants mentioned only that the group had predictable points on their maps in common, like department buildings or nearby coffee houses. 

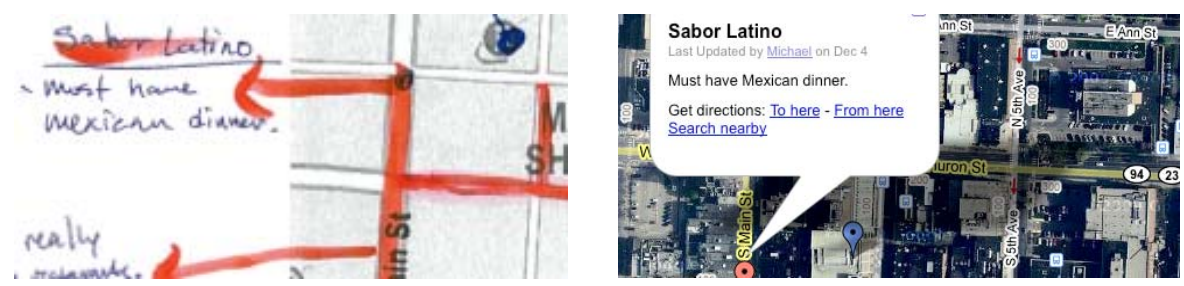

Fig. 3. In phase 4 students compared a map annotated in phase 3 with the same information annotated on a Google map. Students reported an intangible human quality that was missing from the digital Google maps.

During the three-week interval between interviews, each participant intersected with several of the others. Still, most could not recall talking with them about the content of the maps.

In a few cases, the maps revealed new information to one participant about another, but this did not inspire map-related social interaction between the individuals. Instead, participants said the maps simply reinforced their existing impression of the authors:

[p9.06] knows a lot about the area specifically but also since he's been here for a while he knows a lot about places I wouldn't know and he's just a quirky guy, so that the places he chose to mark kind of reflect his personality in a way. (p4.10)

When the maps did diverge from a reader's impression of the author, readers enjoyed learning new things about familiar colleagues. However, several said that this did not affect their overall impression of the person nor their social behavior toward them, because that the maps were no match for their existing knowledge.

So me seeing their maps doesn't really influence...I'm not like all of a sudden, "oh yeah now I want to hang out with [p4.01] because he goes to Bar Louie." I already want to hang out with [p4.01] because I already know his personality. (p4.10)

Many of the phase 3 and 4 participants felt that the maps would be more useful to people who were new to each other, or new to an area, such as when they were starting their graduate program.

\section{Discussion}

Our works sheds light on the factors that influence how people choose to annotate shared maps, and how they read those annotations to learn more about the shared physical environment represented in those maps. While we expected to see that map annotation sharing in a known social group would impact the social interactions among the members of that group, we did not see much evidence for this. Rather, we saw evidence that readers attempt to employ knowledge (or conjecture) about the annotator's personality as a means to interpret the map rather than using the map as a way to influence their view of the author's personality. In other areas, however, we believe that our findings have the potential to guide future systems that depend on shared geospatial annotations. 


\subsection{Implications for Design}

We have described a number of observations that could impact the design of collaborative geospatial annotation systems. Here we summarize those observations and identify the tradeoffs that will face future designers in this domain.

Foster a mix of the familiar and the novel. Determining the credibility of an author is a critical step in a reader's interpretation of the value of a set of annotations. In our study, this was primarily done through calibration: the reader would probe the author's opinion of a subset of familiar places-if the author's judgment matched their own, they would be receptive to novel items included by that author. However, some authors were disinclined to annotate places they thought would be "obvious" to others. Since readers value novel content, but need familiar content to assess an author's opinion of it, application should encourage the annotation of both.

Support the feedback loop between authors and audience. Following the previous point, it is important for authors to be able to assess what content is valuable to their audience, and for the audience to be able to communicate their judgments to authors. Authors adopted strategies for content selection and styles for annotation from others' work, indicating that a dialogue is valuable for guiding authoring activities. Authors also gave considerable thought to the value that would be assessed by their audience, even when that audience was vague. In the absence of established norms and protocols, it is especially important to foster a dialog between annotators and readers, so that they may co-create a mutually-useful annotation style over time.

Understand the appropriate tradeoff between flexibility and aggregatability for a given application. In our study, participants generated annotations by creating freeform marks on paper, a method which afforded extreme flexibility that would be nearly impossible to replicate in an electronic system. At the other end of the spectrum, digital annotation systems using specific points in a highly-structured geospatial coordinate system, provide little stylistic flexibility but allow for the aggregation of many authors' inputs. We encountered shortcomings in both approaches. Hand-drawn annotations were sometimes problematic-writing was hard to read, maps became cluttered, and stylistic choices were deemed arbitrary or even detrimental. However, in some cases, the lack of constraints helped readers better assess authors' personality and credibility: thoughtfulness and thoroughness were imputed to authors based on their stylistic choices, and users expressed difficulty perceiving differences between authors in a more regimented representation. In addition, certain types of flexible annotations were valued by our participants, such as imprecisely bounded areas, routes, or locations whose precise geospatial anchor was unknown. Designers should be aware of these trade-offs for their particular applications and audiences.

\section{Reflect authors' "personality" to aid audience members' interpretation of anno-} tations. In Phases 3 and 4, participants used prior knowledge of authors' personalities to interpret their maps. In Phases 1 and 2, participants attempted to form an idea of the author's personality in order to assess map quality. In cases where an external relationship exists, simply providing a link between a set of annotations and an author's identity would likely suffice. However, where the relationship among authors and audience is primarily or entirely mediated by the annotation system, it becomes 
more important that readers see as much of the original annotation content and personal style of the author as possible.

Maintain high information density. In addition to allowing audience members to quickly assess the interests, tastes, and diligence of an author, viewing a large amount of an author's geospatial annotations in one glance allows them to perceive a coherent "story" of the author's experience of the mapped region, rather than discrete endorsements or critiques. This allows authors to more fully construct the "message" they are communicating to audience members, while also allowing audience members to calibrate the general opinions and biases of a particular author.

\section{Conclusion}

In recent years there has been an increasing number of systems that support authoring and sharing geospatial annotations. As this trend continues, the types of systems, users, and uses will expand ways that are difficult to foresee. The fundamental acts of annotating, sharing, and interpreting maps, however, can be expected to be a central part of any future developments. In this paper, we presented a study of map annotation, which illuminated a number of factors that influence how people decide what to annotate and how they interpret what others' have annotated. We also generated a number of implications for the design of future systems in this space.

We see many possible future directions for this work. One such direction would involve the implementation, deployment, and study of specific applications and/or tools to support map annotation and sharing. It would also be interesting to compare our findings to a study of larger annotation sharing communities such as Google Earth. Given the abbreviated feedback cycle between authors and readers represented in our study, we would be interested to learn more about how annotation practices evolve over time in other communities. We are also interested in exploring how the annotation tools themselves might affect the choices made by authors and the interpretations made by readers, specifically to explore the tradeoff between flexibility and aggregatability discussed in our design implications.

While we see the results of our study as informative in themselves, we are even more hopeful that this work opens new avenues of research for ourselves and others.

Acknowledgments. We would like to thank our study participants, Adam Steenwyk, Mouly Kumaraswamy, Sean Munson, Katherine Connors and Joel Frank. This work supported in part by the National Science Foundation (IIS 0705672).

\section{References}

1. Ackerman, M.S.: Augmenting organizational memory: a field study of Answer Garden. ACM Trans. Inf. Syst. 16(3), 203-224 (1998)

2. Ames, M., Naaman, M.: Why we tag: motivations for annotation in mobile and online media. In: Proceedings of the SIGCHI conference on Human factors in computing systems. ACM, San Jose (2007) 
3. Barkhuus, L., Brown, B., Bell, M., Sherwood, S., Hall, M., Chalmers, M.: From awareness to repartee: sharing location within social groups. In: Proceeding of the twenty-sixth annual SIGCHI conference on Human factors in computing systems. ACM, Florence (2008)

4. Burrell, J., Gay, G.: E-graffiti: Evaluating real-world use of a context-aware system. Interacting with Computers 14, 301-312 (2002)

5. Carter, S., Churchill, E., Denoue, L., Helfman, J., Nelson, L.: Digital graffiti: public annotation of multimedia content. In: CHI 2004 extended abstracts on Human factors in computing systems. ACM, Vienna (2004)

6. Churchill, E., Goodman, E.S.: Mapchat: conversing in place. In: CHI 2008 extended abstracts on Human factors in computing systems. ACM, Florence (2008)

7. Espinoza, F., Persson, P., Sandin, A., Nystr, H., Cacciatore, E., Bylund, M.: GeoNotes: Social and Navigational Aspects of Location-Based Information Systems. In: Proceedings of the 3rd international conference on Ubiquitous Computing, Atlanta, Georgia, USA. Springer, Heidelberg (2001)

8. Goffman, E.: On Face-Work, in Interaction Ritual. Anchor Books (1963)

9. Jones, Q., Grandhi, S.A., Whittaker, S., Chivakula, K., Terveen, L.: Putting systems into place: a qualitative study of design requirements for location-aware community systems. In: Jones, Q., Grandhi, S.A., Whittaker, S., Chivakula, K., Terveen, L. (eds.) Proceedings of the 2004 ACM conference on Computer supported cooperative work. ACM, Chicago (2004)

10. Ludford, P.J., Priedhorsky, R., Reily, K., Terveen, L.: Capturing, sharing, and using local place information. In: Proceedings of the SIGCHI conference on Human factors in computing systems. ACM, New York (2007)

11. Marshall, C.C.: Toward an ecology of hypertext annotation. In: Proceedings of the ninth ACM conference on Hypertext and hypermedia: links, objects, time and space-structure in hypermedia systems: links, objects, time and space—structure in hypermedia systems. ACM, Pittsburgh (1998)

12. Marshall, C.C., Brush, A.J.B.: Exploring the relationship between personal and public annotations. In: Proceedings of the 4th ACM/IEEE-CS joint conference on Digital libraries, ACM, Tuscon (2004)

13. Nelson, L., Churchill, E.F.: Mapmail: restructuring an email client for use in distributed teams. In: CHI 2007 extended abstracts on Human factors in computing systems. ACM, New York (2007)

14. Priedhorsky, R., Terveen, L.: The computational geowiki: what, why, and how. In: Proceedings of the ACM 2008 conference on Computer supported cooperative work, ACM, San Diego (2008)

15. Want, R., Hopper, A., Falc, V., Gibbons, J.: The active badge location system. ACM Trans. Inf. Syst. 10(1), 91-102 (1992) 